\title{
Enhanced antitumor activity of epirubicin combined with cerulenin in osteosarcoma
}

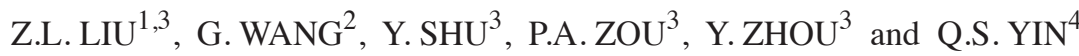 \\ ${ }^{1}$ Graduate School of Southern Medical University, Guangzhou, Guangdong; ${ }^{2}$ Department of Orthopedics, \\ Third Affiliated Hospital of Nanchang University; ${ }^{3}$ Department of Orthopedics, \\ First Affiliated Hospital of Nanchang University, Nanchang, Jiangxi; ${ }^{4}$ Guangzhou General Hospital \\ of Guangzhou Military Region, Guangzhou, Guangdong, P.R. China
}

Received August 16,2011; Accepted October 21, 2011

DOI: $10.3892 / \mathrm{mmr} .2011 .661$

\begin{abstract}
Osteosarcoma (OS) is the most common primary malignant neoplasm in children and adolescents. Epirubicin is one of the chemotherapeutic agents currently used for the treatment of OS; however, the efficacy of chemotherapy is hampered by the acquired drug resistance of OS. Cerulenin, an inhibitor of fatty acid synthase, has been defined as a candidate tumor suppressor. In this study, we explored the combined effect of cerulenin plus the chemotherapy drug, epirubicin, on human OS U2-OS cells in vitro and in vivo. We demonstrated that cerulenin plus epirubicin induced synergistic growth inhibition and enhanced apoptosis in U2-OS cells. We also demonstrated that cerulenin plus epirubicin synergistically suppressed tumor growth in subcutaneously xenografted U2-OS cells in athymic nude mice. Our results indicate that cerulenin enhances the anti-OS effects of epirubicin in vivo and in vitro.
\end{abstract}

\section{Introduction}

Osteosarcoma (OS) is the most common primary malignant neoplasm in children and adolescents. The estimated incidence rate worldwide is $4 /$ million/year, with a peak incidence at the age of 15-19 years (1). By combining surgery with multiagent chemotherapy over the past three decades, the 5-year cumulative survival rate of primary OS patients has been significantly improved to $60-90 \%$ (2-8). Unfortunately, the efficacy of chemotherapy is hampered by acquired drug resistance (9). It is estimated that less than $30 \%$ of patients with recurrent disease are cured (10-12). Therefore, strategies on how to improve the key effect of chemotherapy is the focus for treating OS.

Correspondence to: Dr Q.S. Yin, Guangzhou General Hospital of Guangzhou Military Region, Guangzhou, Guangdong, P.R. China E-mail: yingqs0905@163.com

Professor Y. Shu, Department of Orthopedics, First Affiliated Hospital of Nanchang University, Nanchang, Jiangxi, P.R. China

E-mail: shuyong57@163.com

Key words: cerulenin, epirubicin, combination, antitumor, osteosarcoma
Epirubicin is one of the chemotherapeutic agents used for the treatment of OS $(13,14)$. Epirubicin exhibits growth inhibition of tumors by inducing apoptosis. Conversely, it reduces apoptosis in OS cells by activating NF- $\mathrm{BB}(15)$. At the same time, many other factors also result in the limitation of epirubicin in clinical application, including toxicity and adverse reactions. Fortunately, adjunctive therapies of drugs sometimes reduce adverse reactions and drug resistance. Therefore, we attempted to find a compound, which would enhance the effect of epirubicin against OS.

Cerulenin, a small-molecule fatty acid synthase (FASN) inhibitor, has been isolated from Cephalosporium caerulens. Cerulenin contains an epoxy group that reacts with the ketoacyl synthase domain of FASN. It is one of the first compounds to be found to inhibit FASN in breast cancer cell lines. Cerulenin has revealed significant antitumor activity in many malignant neoplasm cells by inducing apoptosis and growth inhibition (16-19).

In the present study, we investigated the enhanced antitumor activity of epirubicin combined with cerulenin in vitro and in vivo. We observed that there was increased apoptosis and growth inhibition in U2-OS OS cells when treated with cerulenin plus epirubicin in vitro. In addition, the size and weight of U2-OS OS subcutaneously xenografted tumors in athymic nude mice treated with cerulenin plus epirubicin were reduced compared to those treated with either drug alone. Further investigations are required to explore the mechanism of the enhanced effect of cerulenin plus epirubicin.

\section{Materials and methods}

Cell growth assay. A U2-OS cell line was cultured in 96-well tissue culture plates at a density of 5,000 cells/well in minimum essential medium (MEM) containing $10 \%$ fetal bovine serum and $2 \mathrm{mM}$ L-glutamine. Following attachment overnight, the medium was replaced and cells were incubated with increasing concentrations $(2.5,5,10$ and $20 \mu \mathrm{g} / \mathrm{ml})$ of cerulenin (Sigma, St. Louis, MO, USA), epirubicin (Zhejiang Hisun Pharmaceutical Co., China) or combined at different ratios (cerulenin:epirubicin $=1: 1,1: 2$ and 2:1). Following treatment for 48 h, 3-(4,5-dimethylthiazol-2-yl)-2,5-diphenyltetrazolium bromide (MTT) assays were carried out at $490 \mathrm{~nm}$ wavelength in triplicate. The inhibi- 
tion ratio by cerulenin, epirubicin or the combination at each concentration was calculated, and the concentration-viability curves were fitted by Originpro7.5 program. The half maximal inhibitory concentration $\left(\mathrm{IC}_{50}\right)$ values of cerulenin, epirubicin or a combination were determined. All experiments were repeated three times. Interaction between epirubicin and cerulenin was assessed using the combination index $(\mathrm{CI})$ : $\mathrm{CI}=\mathrm{C}_{\mathrm{AC}} / \mathrm{C}_{\mathrm{AS}}+\mathrm{C}_{\mathrm{BC}} /$ $\mathrm{C}_{\mathrm{BS}}$, where $\mathrm{C}_{\mathrm{AC}}$ and $\mathrm{C}_{\mathrm{BC}}$ are the concentration of cerulenin and epirubicin, respectively, in a combined model when the inhibition ratio is $50 \% . \mathrm{C}_{\mathrm{AS}}$ and $\mathrm{C}_{\mathrm{BS}}$ are the concentration of cerulenin and epirubicin, respectively, when used solely at $50 \%$ inhibition. When $\mathrm{CI}$ is $<0.95$, there is a synergistic effect between cerulenin and epirubicin. When $\mathrm{CI}$ is $>1.05$, there is an antagonizing effect between them. When CI is $<1.05$ but $>0.95$, there is an additive effect between them.

Fluorescence-activated cell sorting (FACS). U2-OS cells in exponential growth phase were treated with varying concentrations of cerulenin, epirubicin or the combination for $24 \mathrm{~h}$. Cells were then fixed with $70 \%$ ethanol and stained with Propidium Iodide (PI). FACS analysis was performed to determine the percentage of apoptotic cells and cell cycle distribution by using the EPICS XL flow cytometer and System II software. Jin's Q value, which is also used to assess the adjunctive therapy, was determined by the following: $\mathrm{Q}=$ $\mathrm{E}_{(\mathrm{A}+\mathrm{B})} /\left[\mathrm{E}_{\mathrm{A}}+\left(1-\mathrm{E}_{\mathrm{A}}\right) \times \mathrm{E}_{\mathrm{B}}\right] ; \mathrm{E}_{(\mathrm{A}+\mathrm{B})}$ was the apoptotic percentage at various concentrations when combined, while $E_{A}$ and $E_{B}$ were those when cerulenin and epirubicin were used solely. When the Q-value is $<0.85$, there is an antagonizing effect between cerulenin and epirubicin. When the $\mathrm{Q}$-value is $>1.15$, there is a synergistic effect between them. If the Q-value is $<1.15$ but $>0.85$, there is an additive effect between them.

Evaluation of cerulenin, epirubicin or combination in vivo. BALB/C nude mice (SPF grade, males) aged between 4 and 5 weeks, were obtained from the Shanghai Institute of Materia Medica (Shanghai, China), housed in sterile cages under laminar airflow hoods in a specific pathogen-free room with a 12-h light and 12-h dark schedule and fed autoclaved chow and water ad libitum. All animal procedures and maintenance were conducted in accordance with the institutional guidelines of the University of Nan Chang. Mice were injected with a 0.2-ml suspension containing $2 \times 10^{6} \mathrm{U} 2-\mathrm{OS}$ cells. After 7 days, when tumors reached a volume of $0.1 \mathrm{~cm}^{3}, 24$ mice were randomly separated into four groups as follows: the control group which received a i.p. administration of $0.2 \mathrm{ml}$ phosphate-buffered saline (PBS) containing 10\% dimethyl sulfoxide (DMSO) (group A); the cerulenin group (group B) which received cerulenin treatment $(40 \mathrm{mg} / \mathrm{kg}$ weight); the epirubicin group (group C) which received epirubicin treatment $(2 \mathrm{mg} / \mathrm{kg}$ weight); the cerulenin combined with epirubicin group (group D) which received cerulenin (40 $\mathrm{mg} / \mathrm{kg}$ weight) combined with epirubicin ( $2 \mathrm{mg} / \mathrm{kg}$ weight $)$ treatment. All animals received i.p. administration once every $48 \mathrm{~h}$ which lasted for 12 days. A total of 18 days prior to the end of administration, the mice were sacrificed and the tumors were flaked off. The volume and weight of tumors were measured. The volume and weight inhibition ratios were calculated as follows: $\%$ volume inhibition $=100 \times\left(1-\right.$ volume $_{\text {treated }} /$ volume $\left._{\text {control }}\right) ; \%$ weight inhibition $=100 \times\left(1-\right.$ weight $_{\text {treated }} /$ weight $\left._{\text {control }}\right)$.
Terminal deoxynucleotidyl transferase dUTP nick end labeling (TUNEL) assays. Tumor tissues were cut into slices, which were then paraffin-embedded, followed by TUNEL assays using In Situ Cell Death Detection kit (Roche Diagnostics Corp., Indianapolis, IL, USA). Briefly, following deparaffinization and rehydration, tissue sections were pretreated with sodium chloride-sodium citrate buffer ( $\mathrm{pH} 7.0$ ) at $80^{\circ} \mathrm{C}$ for $20 \mathrm{~min}$, followed by thorough washing in distilled water. Subsequently, sections were digested with Proteinase K for $1 \mathrm{~h}$ with gentle agitation at $37^{\circ} \mathrm{C}$ and digestion was discontinued by washing in running water. Tissue sections were then incubated with fluorescein-labeled deoxyuridine triphosphate (dUTP) reagent and TUNEL reagent in order, according to the manual instructions. Subsequently, the sections were stained with diaminobenzidine (DAB) via horseradish peroxidase (HRP)-conjugated fluorescein antibody and counterstained with $0.5 \%$ methyl green. The sections were mounted with coverslips and imaged; brown-stained cells were considered to be apoptotic cells, and the number of brown-stained cells was used to determine the apoptotic index (AI) by dividing this value by the total number of tumor cells.

Statistical analysis. Data were expressed as the means \pm standard deviation (SD). A one-way ANOVA and least significant difference (LSD) tests were used for statistical analysis, and a p-value of $<0.05$ was considered to be statistically significant. All analyses were performed using SPSS version 13.0 (Statistical Software for Social Sciences, Inc., Chicago, IL, USA).

\section{Results}

Synergistic antitumor effect between cerulenin and epirubicin. Epirubicin is a type of antitumor agent that is widely used; at the same time, cerulenin inhibits cell growth in many types of cancer cell lines. We examined the effect of cerulenin and epirubicin used alone on the growth of the U2-OS cell line, as well as the adjunctive therapy between them, by MTT assay. The growth curves indicated that U2-OS cells were sensitive to both cerulenin and epirubicin, and that the growth inhibition occurred in a dose-dependent manner (Fig. 1A). The $\mathrm{IC}_{50}$ values of epirubicin and cerulenin for U2-OS cells were 9.85 and $22.63 \mu \mathrm{g} / \mathrm{ml}$, respectively. When these two reagents were applied on U2-OS cells adjunctively in the proportions of $1: 1,1: 2$ and $2: 1$ (Fig. 1B), the $\mathrm{IC}_{50}$ values were $8.01,11.54$ and $11.85 \mu \mathrm{g} / \mathrm{ml}$, respectively. All CIs of $0.51,0.58$ and 0.73 in proportions of $1: 1,1: 2$ and $2: 1$, respectively, were $<0.95$, indicated that a synergistic antitumor effect existed between epirubicin and cerulenin.

Epirubicin, cerulenin and combined treatment induces apoptosis in U2-OS cells. FACS analysis was used to examine the mechanism of cell growth inhibition by epirubicin, cerulenin and the combination treatment. Reagents of varying concentrations were added to U2-OS cell cultures in the exponential growth phase. Following treatment for $24 \mathrm{~h}$, treated and untreated cell samples were taken and fixed for FACS analysis. FACS analysis revealed that apoptosis occurred in a dose-dependent manner, regardless of whether cells were treated with epirubicin, cerulenin or a combination (Fig. 2). The percentage of apoptotic cells found in the cells treated 


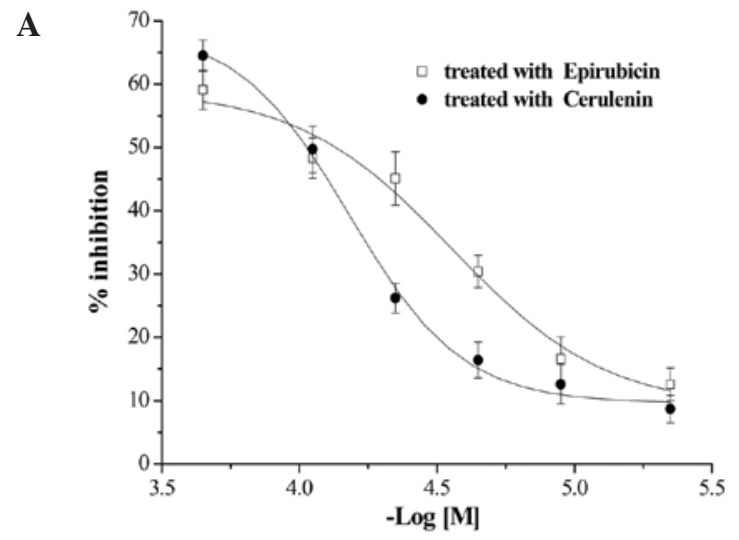

B

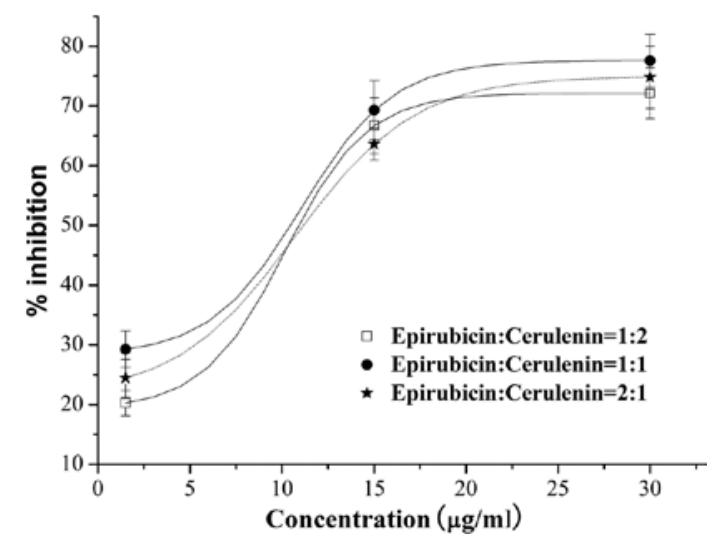

Figure 1. Inhibitory effect of epirubicin, cerulenin or combination treatment on the growth of U2-OS cells. (A) Epirubicin and cerulenin both inhibited U2-OS cell growth in a dose-dependent manner. (B) Epirubicin was applied to U2-OS cells adjunctively with cerulenin in a proportion of 1:1, 1:2 and 2:1. The adjunctive treatment also inhibited U2-OS cell growth in a dose-dependent manner.

with epirubicin was significantly higher than that in cells treated with cerulenin at the same concentration (Table I); 17.27, 26.07 and $31.54 \%$ in cells treated with 1,5 and $10 \mu \mathrm{g}$ / ml epirubicin, respectively. When these two reagents were applied to U2-OS cells in adjunct, the apoptotic percentage improved. In particular, when $1 \mu \mathrm{g} / \mathrm{ml}$ epirubicin was applied to cells in adjunct with $1 \mu \mathrm{g} / \mathrm{ml}$ cerulenin, the Q-value was 1.17 , indicating a synergistic effect between them. Of note, the apoptotic percentage in cells treated by $10 \mu \mathrm{g} / \mathrm{ml}$ of epirubicin and $5 \mu \mathrm{g} / \mathrm{ml}$ of cerulenin in adjunct was only slightly higher than that in cells treated with $10 \mu \mathrm{g} / \mathrm{ml}$ epirubicin alone, with a Q-value of 0.97 .

Epirubicin combined with cerulenin significantly inhibits the growth of xenografted tumors. A significant in vivo antitumor effect was also observed in mice treated with epirubicin combined with cerulenin (Fig. 3). The diameter of the tumor tissue was $>2 \mathrm{~cm}$ in the control group, while in the groups treated with epirubicin and cerulenin the diameter was only $\sim 1.5 \mathrm{~cm}$, with volume inhibition ratios of 36.2 and $34.3 \%$, respectively. However, when the mice were treated with epirubicin combined with cerulenin, the diameter of the tumor tissue was only $\sim 1 \mathrm{~cm}$, with a volume inhibition ratio of $75.1 \%$ compared to the control group. Accordingly, the tumor weights decreased with the treatment of epirubicin, cerulenin and combination treatment, with tumor weight inhibition rates of 35.3, 33.0 and
Table I. Percentage of apoptotic cells in cells treated with epirubicin, cerulenin and their combination.

\begin{tabular}{lll}
\hline Groups & $\begin{array}{c}\text { Percentage of } \\
\text { apoptotic cells }\end{array}$ & Q-value \\
\hline
\end{tabular}

Epirubicin treatment
$1 \mu \mathrm{g} / \mathrm{ml}$
$17.27 \pm 2.34$
$5 \mu \mathrm{g} / \mathrm{ml}$
$26.07 \pm 3.17$
$10 \mu \mathrm{g} / \mathrm{ml}$
$31.54 \pm 1.33$

Cerulenin treatment
$1 \mu \mathrm{g} / \mathrm{ml}$
$7.32 \pm 0.98$
$5 \mu \mathrm{g} / \mathrm{ml}$
$11.17 \pm 1.22$
$20 \mu \mathrm{g} / \mathrm{ml}$
$38.78 \pm 2.13$

Combination treatment

with epirubicin plus

cerulenin

$\begin{array}{ll}1 \mu \mathrm{g} / \mathrm{ml} \text { epirubicin }+ & 26.94 \pm 1.65 \\ 1 \mu \mathrm{g} / \mathrm{ml} \text { cerulenin } & \\ 10 \mu \mathrm{g} / \mathrm{ml} \text { epirubicin }+ & 38.77 \pm 3.46 \\ 5 \mu \mathrm{g} / \mathrm{ml} \text { cerulenin } & \\ 10 \mu \mathrm{g} / \mathrm{ml} \text { epirubicin }+ & 66.19 \pm 3.97 \\ 20 \mu \mathrm{g} / \mathrm{ml} \text { cerulenin } & \end{array}$

${ }^{a}$ Values expressed as the mean \pm standard deviation (SD).

$75.9 \%$, respectively. There was no significant difference in volume and weight inhibition ratios between groups treated with epirubicin and cerulenin alone. However, when epirubicin was applied conjunctively with cerulenin in mice, both volume and weight inhibition ratios increased significantly. The $\mathrm{Q}_{\text {weight }}$ and $\mathrm{Q}_{\text {volume }}$ were 1.32 and 1.34, respectively, which indicated that a synergistic antitumor effect existed between epirubicin and cerulenin.

Epirubicin combined with cerulenin significantly induces apoptosis of tumor cells. The fact that epirubicin combined with cerulenin significantly inhibited the growth of xenografted tumors raised the possibility that adjunctive therapy may greatly induce apoptosis in tumor cells. TUNEL assays were carried out to test this theory. Epirubicin, cerulenin and the combination treatment all induced apoptosis in tumor cells (Fig. 4). As noted in Fig. 4B-D, brown-stained nuclei were evident throughout, whereas little staining was noted in Fig. 4A. The AI in groups A, B, C and D was 3.5 $\pm 1.8,12.1 \pm 6.1$, $12.3 \pm 7.2$ and $20.1 \pm 7.3 \%$, respectively. Although there was no significant difference in AI between groups B and C, the AI in group D increased, which may partly explain the marked increase in volume and weight inhibition ratios when treated with epirubicin combined with cerulenin.

\section{Discussion}

OS is the most common malignant neoplasm of the bone. Owing to the rapid and aggressive nature of the disease, previously, the standard treatment for OS was amputation of the affected limb; however, the cure rate associated with 

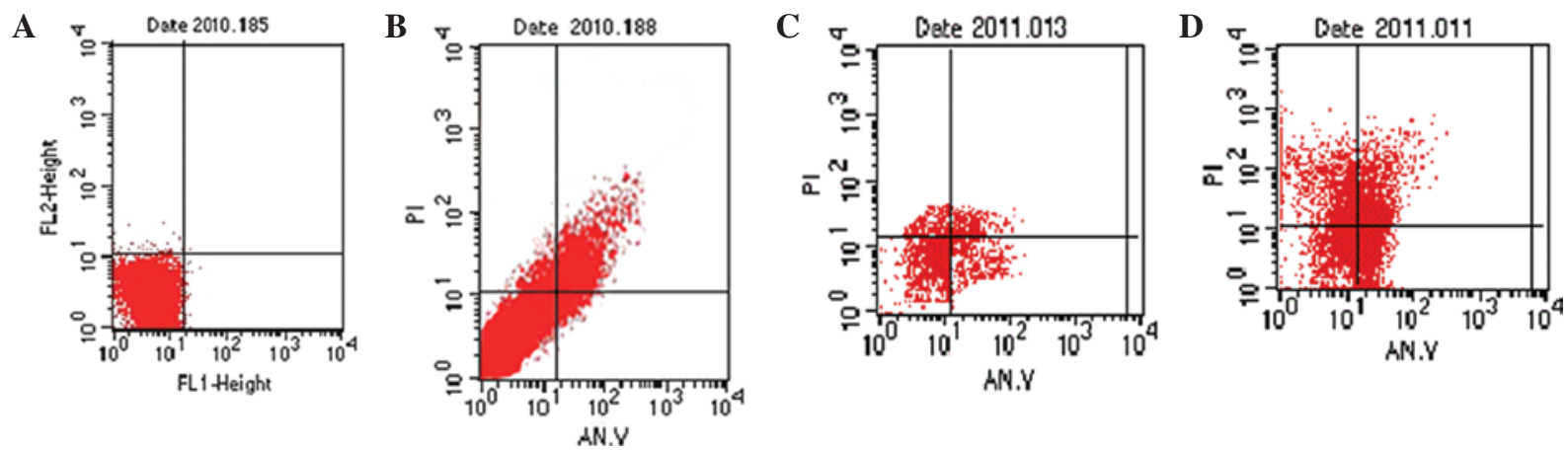

Figure 2. FACS analysis of U2-OS cells treated with epirubicin, cerulenin and the combination treatment for 24 h. (A) Few apoptotic cells were noted in the control group. Yet, the percentage of apoptotic cells was (B) $31.54 \%$ when treated with $10 \mu \mathrm{g} / \mathrm{ml}$ epirubicin and (C) $38.78 \%$ when treated with $20 \mu \mathrm{g} /$ $\mathrm{ml}$ cerulenin. (D) The percentage of apoptotic cells rose to $66.19 \%$ when treated with $10 \mu \mathrm{g} / \mathrm{ml}$ epirubicin in conjunction with $20 \mu \mathrm{g} / \mathrm{ml}$ cerulenin. FACS, fluorescence-activated cell sorting; PI, propidium iodide.
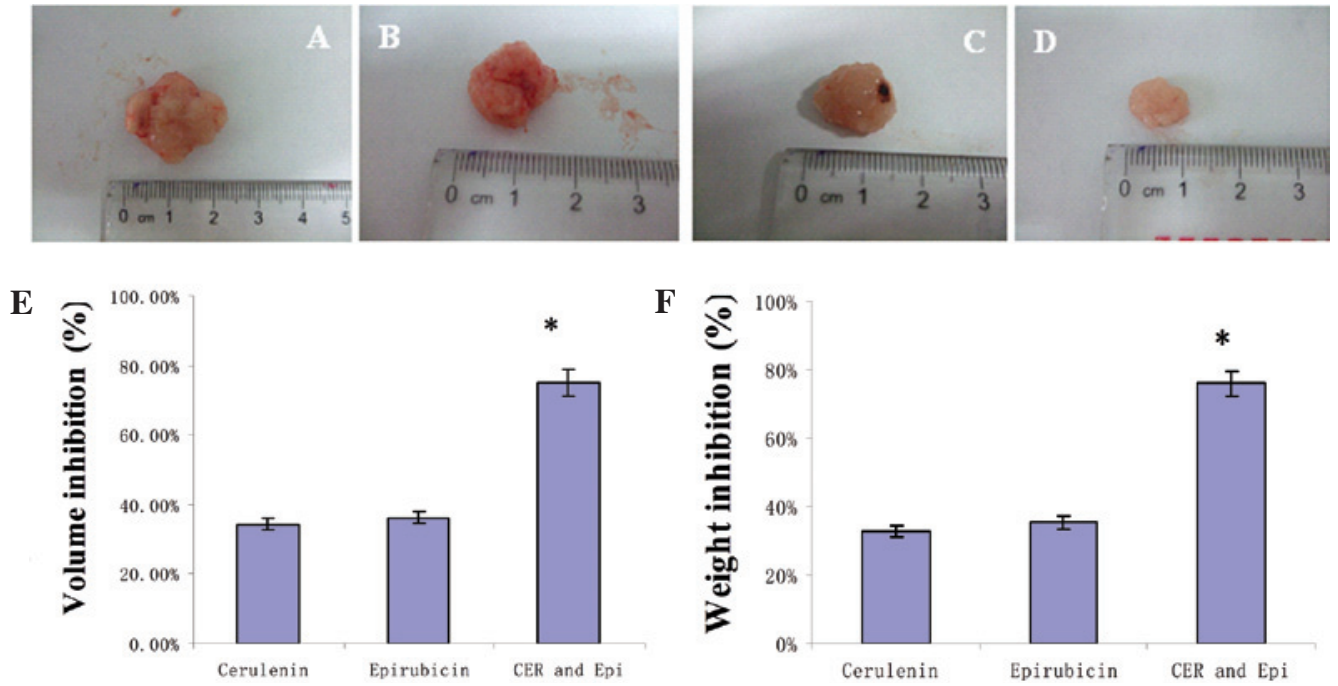

Figure 3. Tumor tissues peeled from xenografted nude mice following treatment with epirubicin, cerulenin and a combination treatment. Representative images are shown for each group. (A) In the control group, the diameter of the tumor tissue was $\sim 2 \mathrm{~cm}$. Following treatment with (B) epirubicin and (C) cerulenin, the diameter of the tumor tissue decreased to $\sim 1.5 \mathrm{~cm}$; concurrently, the weight of the tumor tissue was reduced. (D) When treated with epirubicin combined with cerulenin, the volume and weight of the tumor tissue decreased greatly. (E) The volume inhibition ratio of the cerulenin plus epirubicin group was significantly higher than that of the control, cerulenin and epirubicin alone groups. Columns, mean ( $\mathrm{n}=6)$; bars, $\mathrm{SD}$. " $\mathrm{P}<0.05$ vs. control; cerulenin and epirubicin groups. (F) The inhibition ratio of group D was significantly higher compared to the cerulenin and epirubicin groups. Columns, mean ( $\mathrm{n}=6$ ); bars, SE. "P<0.05 vs. cerulenin and epirubicin groups. SD, standard deviation; Epi, epirubicin; SE, standard error; CER, cerulenin.

A

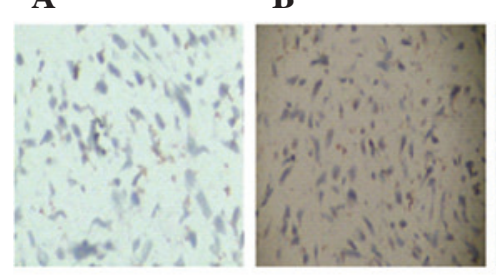

C

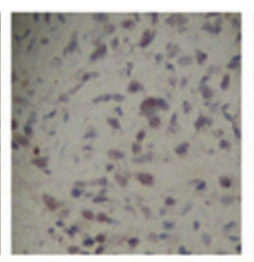

D

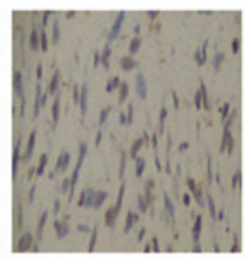

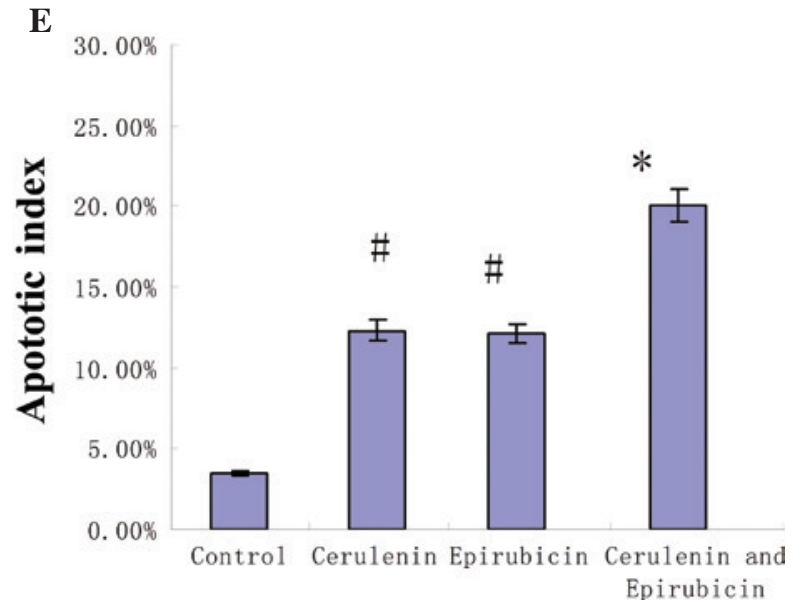

Figure 4. TUNEL assays of tumor tissue slides. (A-D) Representative images for each group. (A) Tumor-bearing mice received i.p. administration of $0.2 \mathrm{ml}$ PBS and tumor tissue slides were prepared. TUNEL assays indicated that there were only a few fluorescent cells, with a ratio of $<5 \%$. Brown-stained cells were barely detected. When tumor-bearing mice were treated with (B) cerulenin and (C) epirubicin, the ratio of brown-stained cells increased significantly to $\sim 12 \%$. (D) When epirubicin combined with cerulenin was applied to tumor-bearing mice, there were widespread brown-stained cells, with a ratio of almost $20 \%$. (E) The apoptotic index was significantly higher than that in the control, cerulenin and epirubicin alone groups. Columns, mean ( $\mathrm{n}=6$ ); bars, SD. ${ }^{*} \mathrm{P}<0.05$ vs. control, cerulenin and epirubicin groups. PBS, phosphate-buffered saline; TUNEL, terminal deoxynucleotidyl transferase dUTP nick end labeling; SD, standard deviation. 
this method was less than $10 \%$ and almost all patients died within 1 year from diagnosis. Over the past three decades, the development of effective chemotherapy agents has reduced the incidence of metastasis and mortality $(2,20)$. Chemotherapy is, thus, an important treatment for OS.

Epirubicin, a member of the anthracycline family, is widely applied for the treatment of OS patients, profiting from its weak side effects on the heart. However, the acquired resistance of cancer cells to epirubicin has become a major obstacle for successful cancer therapy (21). Various studies have reported that NF- $\kappa B$ plays an important role in drug resistance (22-24). Cerulenin, an inhibitor of FASN, inhibits the growth of tumor cells by inhibiting NF- $\mathrm{KB}$ and inducing apoptosis (25), which may reverse NF- $\mathrm{KB}$ activation by epirubicin. Therefore, we hypothesized that cerulenin enhances the anti-OS effects of epirubicin. Fortunately, this hypothesis was confirmed both in vitro and in vivo in our study. The in vitro study indicated that when epirubicin was applied in adjunct with cerulenin in proportions of 1:1 and 1:2, there was a synergistic antitumor effect between them, with Q-values greater than 1.15. However, when epirubicin was combined with cerulenin in a proportion of 2:1, there was only an additive effect between them, with a Q-value of 0.97. Our data suggest that cerulenin enhances the epirubicin anti-OS effect in vitro.

In the in vivo study, when epirubicin or cerulenin were applied alone, the volume inhibition ratios were 36.2 and $34.3 \%$, respectively, and the weight inhibition ratios were 35.3 and $33 \%$, respectively. However, when epirubicin was applied in adjunct with cerulenin, there were significant increases in volume and weight inhibition ratios, which were 75.1 and $75.9 \%$, respectively, with $\mathrm{Q}_{\text {weight }}$ and $\mathrm{Q}_{\text {volume }}$ of 1.32 and 1.34, respectively. These data indicate that there were synergistic effects between cerulenin and epirubicin for anti-OS in vivo.

In conclusion, our results indicate that a synergistic or addictive effect exits between cerulenin and epirubicin for anti-OS in vitro and a synergistic effect in vivo. It has been suggested that cerulenin combined with epirubicin may be a potential treatment strategy for OS. However, the combination of cerulenin and epirubicin was administered using three ratios of the agents in vitro, and only one proportion in vivo was applied in this study. Whether another ratio of the agents, in particular increased doses of epirubicin, has enhancing effects between them requires further investigation. In addition, the mechanism involved in the synergistic effect between epirubin and cerulenin remains unknown. In this study, we did not verify that cerulenin reverses NF- $\mathrm{KB}$ activation by epirubicin. Perhaps some other factors contribute to the synergistic antitumor effect between them. Future experiments need to be carried out in order to try to elucidate this mechanism.

\section{Acknowledgements}

This study was supported by a grant from the Education Department of Jiangxi Province, China (grant GJJ09102).

\section{References}

1. Mirabello L, Trosis RJ and Savage SA: Osteosarcoma incidence and survival rates from 1973 to 2004: data from the Surveillance, Epidemiology, and End Results Program. Cancer 115: 1531-1543, 2009.
2. Lewis VO: What's new in musculoskeletal oncology. J Bone Joint Surg Am 89: 1399-1407, 2007.

3. Iwamoto Y, Tanaka K, Isu K, et al: Multiinstitutional phase II study of neoadjuvant chemotherapy for osteosarcoma (NECO study) in Japan: NECO-93J and NECO-95J. J Orthop Sci 14: 397-404, 2009.

4. Meyers PA, Schwartz CL, Krailo M, et al: Osteosarcoma: a randomized, prospective trial of the addition of ifosfamide and/or muramyl tripeptide to cisplatin, doxorubicin, and high-dose methotrexate. J Clin Oncol 23: 2004-2011, 2005.

5. Lee JW, Kim H, Kang HJ, et al: Clinical characteristics and treatment results of pediatric osteosarcoma: the role of high dose chemotherapy with autologous stem cell transplantation. Cancer Res Treat 40: 172-177, 2008.

6. Cho Y, Jung GH, Chung SH, et al: Long-term survivals of stage IIB osteosarcoma: a 20 -year experience in a single institution. Clin Orthop Surg 3: 48-54, 2011.

7. Tsuchiya H, Tomita K, Mori Y, et al: Caffeine-assisted chemotherapy and minimized tumor excision for nonmetastatic osteosarcoma. Anticancer Res 18: 657-666, 1998.

8. Bölling T, Schüller P, Distelmaier B, et al: Perioperative high-dose rate brachytherapy using a bendy applicator (flab): treatment results of 74 patients. Anticancer Res 28: 3885-3890, 2008.

9. Kusuzaki K, Hirata M, Hashiguchi S, et al: Acquisition of multidrug resistance in osteosarcomas, analyzed by doxorubicin binding assay, and histologic response to chemotherapy. Anticancer Res 19: 5159-5162, 1999.

10. Schwartz CL, Gorlick R, Teot L, et al: Multiple drug resistance in osteogenic sarcoma: INT0133 from the Children's Oncology Group. J Clin Oncol 25: 2057-2062, 2007.

11. Chou AJ and Gorlick R: Chemotherapy resistance in osteosarcoma: current challenges and future directions. Expert Rev Anticancer Ther 6: 1075-1085, 2006.

12. Dean M, Fojo T and Bates S: Tumour stem cells and drug resistance. Nat Rev Cancer 5: 275-284, 2005.

13. Ayan I, Kebudi R and Ozger H: Childhood osteosarcoma: multimodal therapy in a single institution Turkish series. Cancer Treat Res 152: 319-338, 2009.

14. Luisi FA, Petrilli AS, Tanaka C, et al: Contribution to the treatment of nausea and emesis induced by chemotherapy in children and adolescents with osteosarcoma. Sao Paulo Med J 124: 61-65, 2006.

15. Das KC and White CW: Activation of NF- $\kappa$ B by antineoplastic agents. Role of protein kinase C. J Biol Chem 272: 14914-14920, 1997.

16. Elbaz A, Wu X, Rivas D, et al: Inhibition of fatty acid biosynthesis prevents adipocyte lipotoxicity on human osteoblasts in vitro. J Cell Mol Med 14: 982-991, 2010.

17. Wang WQ, Zhao XY, Wang HY, et al: Increased fatty acid synthase as a potential therapeutic target in multiple myeloma. J Zhejiang Univ Sci B 9: 441-447, 2008.

18. Okawa Y, Hideshima T, Ikeda H, et al: Fatty acid synthase is a novel therapeutic target in multiple myeloma. Br J Haematol 141: 659-671, 2008.

19. Pizer ES, Jackisch C, Wood FD, et al: Inhibition of fatty acid synthesis induces programmed cell death in human breast cancer cells. Cancer Res 56: 2745-2747, 1996.

20. Cesari M, Alberghini M, Vanel D, et al: Periosteal osteosarcoma: a single-institution experience. Cancer 117: 1731-1735, 2011.

21. Sun WL, Chen J, Wang YP, et al: Autophagy protects breast cancer cells from epirubicin induced apoptosis and facilitates epirubicin-resistance development. Autophagy 1: 7, 2011.

22. Li QQ, Chen ZQ, Cao XX, et al: Involvement of NF- $\mathrm{B} /$ miR-448 regulatory feedback loop in chemotherapy-induced epithelial-mesenchymal transition of breast cancer cells. Cell Death Differ 18: 16-25, 2011.

23. Fu ZY, Lv JH, Ma CY, et al: Tissue inhibitor of metalloproteinase- 1 decreased chemosensitivity of MDA- 435 breast cancer cells to chemotherapeutic drugs through the PI3K/AKT/NF- $\kappa B$ pathway. Biomed Pharmacother 65: 163-167, 2011.

24. Cao W, Zhang Y, Zhang D, et al: Effect of antisense oligodeoxynucleotide directed to NF-kappa B RelA on Bcl-XL mRNA in extended drug resistance leukemia cell line HL-60/E6. Tongji Med Univ 21: 32-34, 2001.

25. Menendez JA, Mehmi I, Atlas E, et al: Novel signaling molecules implicated in tumor-associated fatty acid synthase-dependent breast cancer cell proliferation and survival: role of exogenous dietary fatty acids, p53-p21WAF1/CIP1, ERK1/2 MAPK, p27KIP1, BRCA1, and NF-kB. Int J Oncol 24: 591-608, 2004. 